\title{
Post Processing Method for Lagrangian Spray Field Based on Mie Scattering Theory
}

\author{
Shangning Wang ${ }^{1}$, Jingjing $\mathrm{CaO}^{1}$, Shangze Yang ${ }^{1}$, Xuesong $\mathrm{Li}^{1}$, David L.S. Hung ${ }^{2}$, and Min $\mathrm{Xu}^{* 1}$ \\ ${ }^{1}$ School of Mechanical Engineering, Shanghai Jiao Tong University, Shanghai, China \\ ${ }^{2}$ University of Michigan-Shanghai Jiao Tong University Joint Institute, \\ Shanghai Jiao Tong University, Shanghai, China
}

\begin{abstract}
Numerical simulation and experiment are regarded as the two major methods in the investigation of spray and atomization. Simulation methods major in predicting spray developments, whose model are supposed to be calibrated using corresponding experimental data, but direct comparisons between simulation data and experimental results might lead to confusion when focusing on spray boundaries or penetration, as the light scattering physics during imaging is always likely to be ignored in CFD post processing. In many cases, CFD provides data points that are invisible to human eyes or cameras, resulting in variance in boundary confirming process. Previous studies discussed backlit conditions in Euler-based simulations to identify spray boundaries, but for most commonly used Lagrangian-based simulations, this topic remains undiscussed. In Lagrangian-based methods, droplets are treated as discrete particles, where scattering plays a more crucial role compared with that in Euler fields. In this study, light intensity analysis based on Mie Scattering theory and intensity integration focusing on Lagrangian field has been presented, aiming to present simulation data of spray coincides with Mie Scattering image as much as possible. It is found that particle size and in parcel numbers are related with the scattering intensity of droplet particles, and the calibrated CFD data using Mie-Scattering theory shows a better coherence with Mie Scattering imaging results in tested conditions compared with raw simulation data, and this variance tends to be greater when spray density difference is apparent.
\end{abstract}

\section{Keywords}

CFD data calibration, Mie scattering, Lagrangian spray field

\section{Introduction}

Computer Fluid Dynamics (CFD) method has been one of the main streams in the design and development of internal combustion engine systems, and this trend tends to grow in the upcoming years. By selecting suitable physical models and proper algorithms, CFD could simulate detailed processes of fuel spray development. In order to obtain valid simulation results, physical models must be correlated with real conditions, and for those halfexperimental based models with changing parameters, calibrations must be applied in the first place[1,2], adjusting the variable elements to meet up with real conditions.

One often neglected reason for not satisfying simulation accuracy is the incorrect calibration method. In temporary practice, the calibration of spray characters is mainly based on optical diagnostic experiments, for penetration and spray angle calibrations, often Miescattering or backlit imaging are conducted to obtain macroscopic photographs[3], spray boundaries are adopted using algorithms, and finally penetration and angles are figured out. As is often the case, bias exist between CFD researchers and experimental researchers, and 
none-matching calibration data could often be used as matching ones, resulting in wrong calibrated models.

In CFD simulations, the results faithfully record all spray droplets' locations and other information regardless of its size and visibility, and even if there is only one tiny particle at the far end, simulation results regard it as visible, and calculated the penetration based on mass accumulations or other criteria. In the optical experiments, however, the boundary identification is strongly related with scattering or other light transmitting processes. For particles of certain small sizes, or being separated far away from neighboring particles, the light intensity tends to be low, and such particles are less likely to be recorded by cameras, or are less likely to be estimated as within the threshold of spray boundary judgements, which is a common data processing method in optical diagnostics[4]. Such conditions are evident especially in heated sprays or far end sprays, and this results in a shifting of spray boundaries. If researchers use such shifted boundaries as calibration data, chances are that wrong results would be obtained, as the imaged boundary is not the real boundary we should use as criteria.

It is difficult to reform the invisible drops in imaging results, but by applying optical methodology, it is possible to post process the CFD data to match with the imaged results. Attempts have been made to correct spray angle simulations with backlit imaging data based on Beer-Lambert law[5], and the results have been proved to be helpful for model refinements, but due to the theory difference, such refinement is not proper for Mie-scattering based experiments, which are also widely used in spray detecting. In this research, efforts are made to post-process the CFD data via Mie-scattering theory, and make it 'apple to apple' with experimental images, so as to achieve effective calibration for following studies.

\section{Mie-scattering Based Particle Light Intensity Analysis}

The light intensity distribution of Mie-scattering image obeys the Mie-scattering theory. When the light from light source meets droplets, a part of the light is scattered, and the direction of the scattered light, as well as the intensity distribution is highly related with the shape, size, and number density of the corresponding particle. As the droplets discussed in spray studies normally range from 1-100 micrometers, the scattering phenomenon falls in Miescattering region. There are several factors that mainly governs imaged Mie-scattering particles, including particle size, particle density, and particle sheltering effect.

The governing equations of Mie-scattering of a single, ideal round droplet could be depicted as follows[6]:

$$
\begin{gathered}
I_{s c a}=I_{0} \frac{\lambda^{2}}{8 \pi^{2} r^{2}} I(\theta, \varphi) \\
I(\theta, \varphi)=\left|S_{1}(\theta)\right|^{2} \sin ^{2} \varphi+\left|S_{2}(\theta)\right|^{2} \cos ^{2} \varphi \\
S_{1}(\theta)=\sum_{n=1}^{\infty} \frac{2 n+1}{n(n+1)}\left(a_{n} \pi_{n}+b_{n} \tau_{n}\right) \\
S_{2}(\theta)=\sum_{n=1}^{\infty} \frac{2 n+1}{n(n+1)}\left(a_{n} \tau_{n}+b_{n} \pi_{n}\right) \\
a_{n}=\frac{\varphi_{n}(\alpha) \dot{\varphi}_{n}(m \alpha)-m \varphi_{n}(m \alpha) \dot{\varphi}_{n}(\alpha)}{\varepsilon_{n}(\alpha) \dot{\varphi}_{n}(m \alpha)-m \varepsilon_{n}(\alpha) \varphi_{n}(m \alpha)} \\
b_{n}=\frac{m \varphi_{n}(\alpha) \dot{\varphi}_{n}(m \alpha)-\varphi_{n}(m \alpha) \dot{\varphi}_{n}(\alpha)}{m \varepsilon_{n}(\alpha) \varphi_{n}(m \alpha)-\varphi_{n}(m \alpha) \varphi_{n}(\alpha)}
\end{gathered}
$$




$$
\begin{gathered}
\varphi_{k}(\alpha)=\sum_{m=0}^{\infty} \frac{(-1)^{m}}{m ! \Gamma(m+k+1)}\left(\frac{\alpha}{2}\right)^{2 m+k} \\
\varepsilon_{k}(\alpha)=\frac{\varphi_{-k}(\alpha)-e^{k \pi i} \varphi_{k}(\alpha)}{i \sin (k \pi)}
\end{gathered}
$$

And the descriptions of parameters could be referred in Nomenclature. For practice, the distance difference between particles from the camera could be neglected, therefore $r$ could be treated as constant. For given fuel and ambient, the refractive indexes are constant, so the polarized angle and relative refractive index are also constant. The Legendre functions are only changing with $\alpha$. We assume that the light received by the camera share the same scattering angle of $90^{\circ}$, under such assumptions, the scattering intensity distributions with varying particle diameters could be obtained, and the trend could be depicted in Figure1. This value is highly related with phase function. It should be noted that when particle diameter is no greater than certain threshold, which is related with light wavelength and other physical properties, the intensity distribution could be well fitted in polymetric form:

$$
I \propto D^{2}
$$

This is a commonly accepted equation in some highly cited researches[7,8], but it should be noted that such relation is no longer suitable as particle diameter exceeds certain value, as depicted in Figure1, whose calculation are based on eq(1)-(8) via MiePlot [9], this figure depicts the light intensity distribution of water droplets of different sizes under the light of $650 \mathrm{~nm}$ wavelength in atmosphere, and the polymetric region could not cover the diameter range greater than $20 \mu \mathrm{m}$.

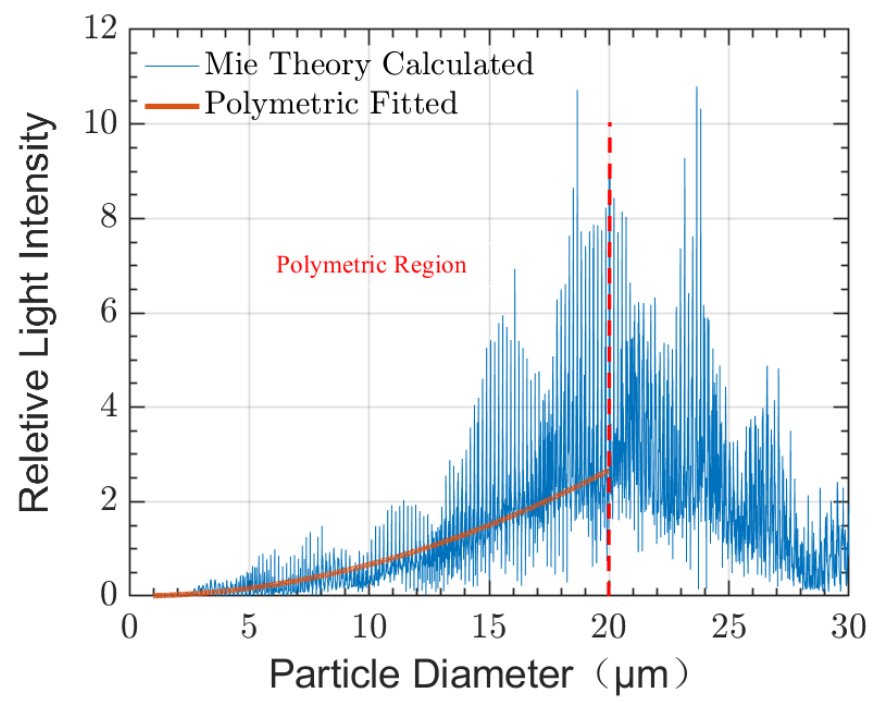

Figure 1. Mie-Scattering Intensity of Single Particle (wave length $=650 \mathrm{~nm}$, isooctane)

In the most applied Lagrangian simulation frame, the droplets with similar size within a nearby region are represented by parcels, and the numbers contained by a parcel is depicted by parcel number density. The light superposition effect could greatly affect the light intensity captured by the camera. Assume the distance difference between particles and camera could be neglected compared with the distance from light source to particles, the phase difference could be neglected, then the light scattered by different particles could be regarded as coherent, then the total light intensity could be depicted as follows:

$$
I^{2}=I_{1}^{2}+I_{2}^{2}+\ldots+I_{n}^{2}+a_{12} I_{1} I_{2}+a_{13} I_{1} I_{3}+\ldots+a_{n(n-1)} I_{n} I_{n-1}
$$

Assume uniform initial intensity, the equation could be treated as: 


$$
I^{2}=I_{0}\left(n+\mathrm{C}_{n}^{2}\right)=I_{0}\left(\frac{1}{2} n^{2}+n\right)
$$

It should be noted that the above effect temporary considers only the superposition effect inside of parcel, the superposition between parcels is not considered. This is because the distance between parcels is greater than that inside of parcel, thus the summation effect is not dominating. However, if the parcel is isolated far away from other parcels, it is more likely to be invisible in camera, therefore, a filter should be added to move away the isolated parcels. When the parcel is at the behind of the spray plume, even if the light from source could enlighten it, the scattered light is not likely to reach the camera, as it could be blocked by the parcels in the front, such effect is regarded as the sheltering effect, and the sheltered parcels contribute a lower light intensity compared with none sheltered ones. It should be noted that multiple scattering effect is not considered in this assumption, if considered, the shelter incident should be modified not only for the behind parcel, but also the parcels around it. In this research, the sheltering coefficient is simplified based on the order of particles sharing approximate $x$ and $y$ values. Further efforts should be made to investigate more reasonable calculations.

To sum up, the combined intensity factor considering size, number density and sheltering could be depicted as:

$$
I^{2} \propto \gamma P(D)\left(\frac{1}{2} n^{2}+n\right)
$$

Where $\gamma$ is the sheltering factor that is less than 1 , and $P(D)$ is the phase function that is determined by particle diameter.

\section{Experimental Setup}

Mie-scattering imaging is a commonly applied optical diagnostic technique in macroscopic spray analysis that could obtain spray penetration and spray angle developments. The basic experimental setup of Mie-scattering is depicted as Figure2. Details could be referred in previous articles[10], and would not be introduced here for brevity. A Delphi single nozzle injector has been applied in this study.

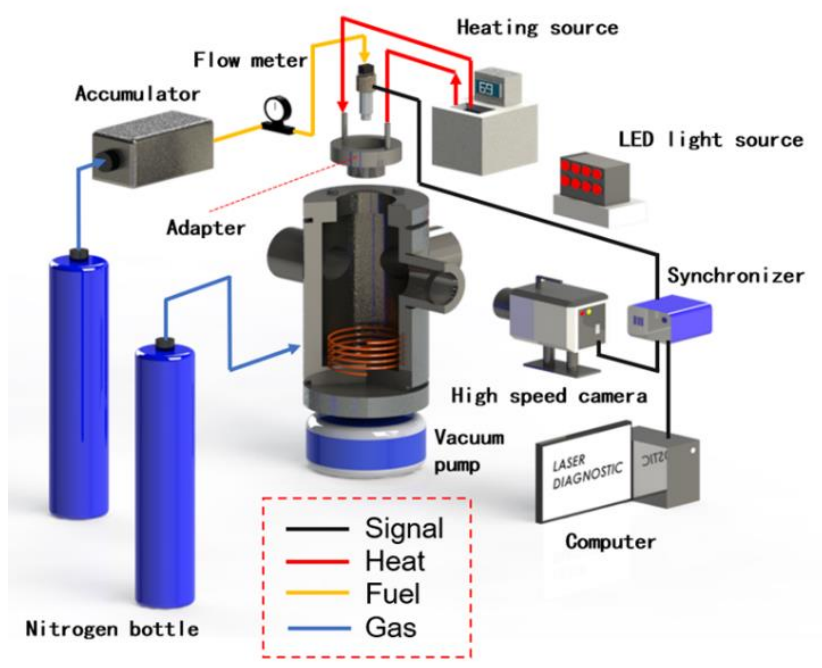

Figure 2. Mie-Scattering Imaging Experimental Setup

In engineering practices, multi cycles of sprays are imaged and averaged so as to denoise the signal and capture main features of spray. It should be noted that the detailed information especially at the edges of spray are tend to be neglected in the averaging process, and it is this averaged data that is used to be the calibration base line of CFD models. Compared with the distance between light source to spray and spray to camera, the distance between 
particles could be neglected in eq(1). Detailed information of this experiment is concluded in Table 1.

Table 1 - Experimental Setup Conditions

\begin{tabular}{cc}
\hline Items & Specifications \\
\hline Fuel type & Isooctane \\
Injection pressure $(\mathrm{MPa})$ & 10 \\
Ambient pressure $(\mathrm{kPa})$ & 100 \\
Fuel temperature $\left({ }^{\circ} \mathrm{C}\right)$ & 20 \\
Injection duration $(\mathrm{ms})$ & 0.5 \\
Light source wavelength $(\mathrm{nm})$ & 650 \\
Repeated test number & 20 \\
Imaging frequency $(\mathrm{kHz})$ & 20 \\
Nozzle L/D & 1.5 \\
Plume angle $\left({ }^{\circ}\right)$ & 0 \\
Flowrate @ 10MPa $(\mathrm{cc} / \mathrm{s})$ & 3.75 \\
\hline
\end{tabular}

\section{Results and Discussions}

The simulation process has been conducted using OpenFOAM5, KH-RT model has been selected as the breakup model, as the test condition is ranged in cold spray region, the most widely accepted parameters are used without further validation[11], and EnSight v10.1 is applied to obtain the particle data. The simulation time step is set to be $1 \mu \mathrm{s}$, and the mesh is of reasonable quality. As the simulation itself is not the focus of this research, default settings for mature spray modeling have been used as much as possible, and detailed information about simulation settings would not be presented here out of brevity.

The data processing procedures are presented in Figure 3 for both experimental results and simulation data. The image of experiment is an average of 20 injection cycles, and the boundary analysis chooses a threshold of $95 \%$ of maximum light intensity according to SAEJ2715 regulations[4]. The simulation results obtained from EnSight provide location, velocity, particle diameter and particle number density. The light intensity of each particle is obtained using eq(12), and the boundary is also obtained based on same threshold.

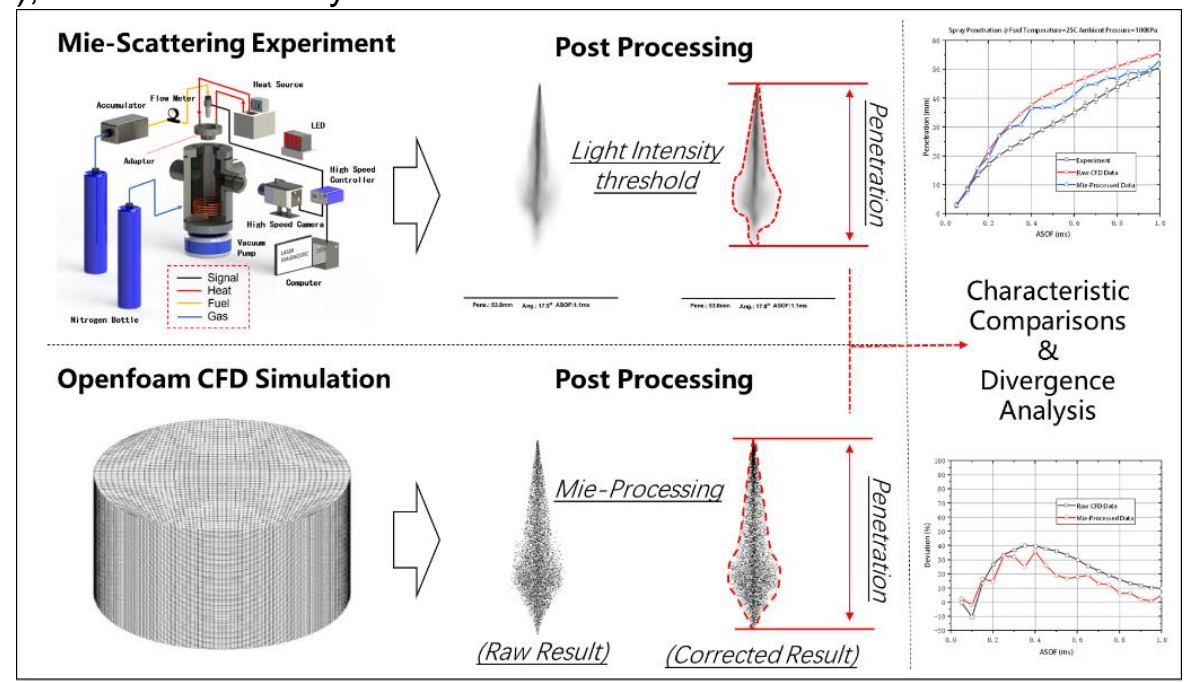

Figure 3. Data Processing for Both Experiment and Simulation

The image of experimental, raw CFD result and correlated CFD result at $1 \mathrm{~ms}$ ASOF are presented in Figure4, and the penetration of experimental data, penetration of raw simulation data (the distance above which accumulated mass fraction is $99 \%$ of total injected fuel), and the penetration of the post-processed Mie-scattering based data are presented in Figure 5, 
while panel (a) gives the comparison of the three penetrations from initial time step to the end of simulation, panel (b) gives the evolution of the standard variation of the two sets of CFD data compared with experimental one.

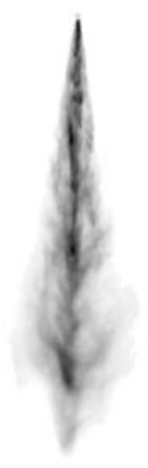

Experiment

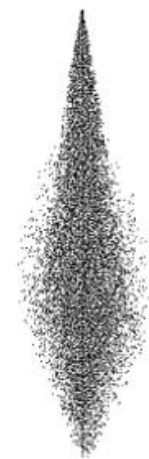

Raw Simulation

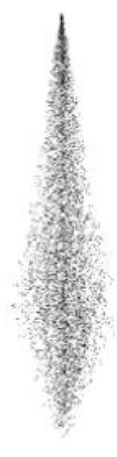

Mie Correlated

Figure 4. Spray Macroscopic Image of Different Means of Processing

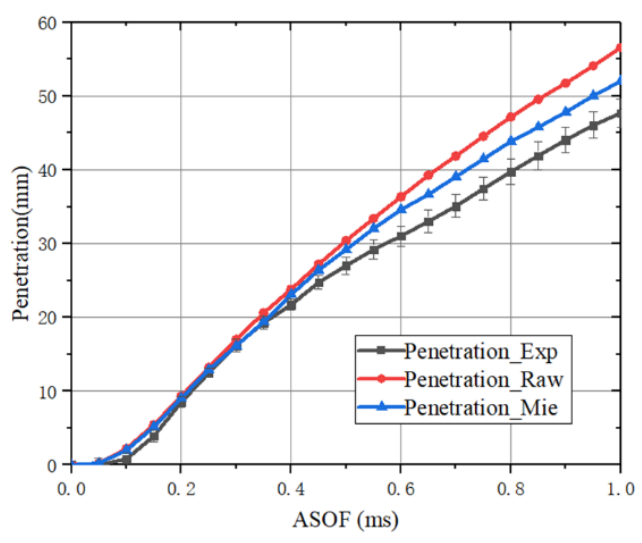

(a) Overall Comparison Result

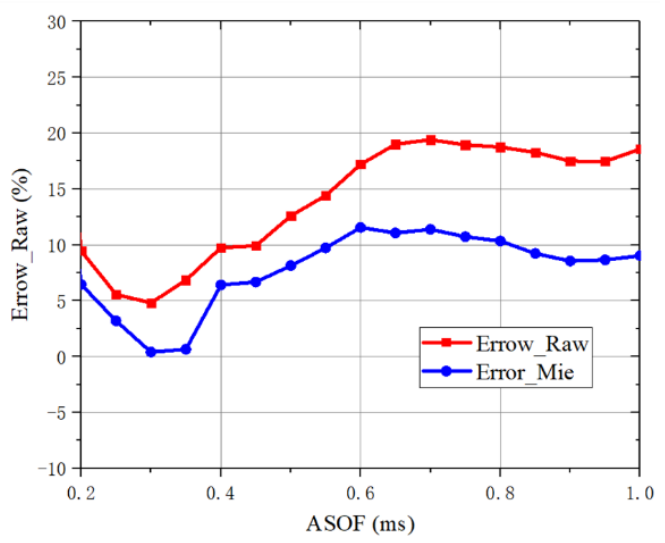

(b) Standard Deviation

Figure 5. Comparison of Spray Penetration Under Test Condition

The results presented in Figure 4 could show that, when considered the correlation, the pattern of the simulated spray would be much more similar with the experimental image, with a relative dense core in the middle, while the surrounding areas are comparably dilute.

It could be depicted from Figure 5 that, the three penetrations share the same trend and is quite close with each other when it comes to value. This is reasonable as the default settings of spray modelling in OpenFOAM is targeted on cold sprays at relatively low injection pressure, the model has been proved efficient for the cold spray condition by many previous researches, and the well-coincide curves at the initial period presented in Figure 4(a) confirms that the simulation as well as experiment conducted in this research is of good quality. Even though the results given show a good agreement of penetration in the end, it should be noted that at the beginning of spray, the deviation between simulation results and experimental results are quite obvious, the experimental curve sees an abrupt acceleration before a relative constant growing speed, this might be the caused by the needle lifting reaction, which is difficult for simulation studies, further studies would choose other injectors to see if this is a common condition or individual result.

In the time range between $0.4 \mathrm{~ms}$ to $1.0 \mathrm{~ms}$ ASOF, the penetration curves share a common trend, and the relative errors have been depicted in Figure 5(b), it could be seen that, under 
the test condition, during the targeted time range, the error between raw simulation result and experiment is within $20 \%$, which is of quite good accuracy, as for the correlated data, the error could be lowered to no more than $10 \%$, and remains almost constant when the spray is fully developed. Such a result proves that the correlation method presented in this study is of good importance, and the deviation related to the difference between imaging and simulation could be eliminated using the Mie theory-based correlations.

It should be noted that due to the limitation of the phase-change spray simulation theory, it is difficult to apply such calibration to the high temperature fuels like flash-boiling sprays, but it is such extreme conditions that creates an obvious density variation between different regions of the spray, and would be in more desperate need for experimental to simulation calibration compared with sub-cooled spray mentioned in this research. The authors would continue focusing on the progress of simulation methods for sprays, and validate the necessity of the Mie-Scattering calibration in wider range of test conditions.

\section{Conclusions}

In this research, a Mie-Scattering based spray simulation data calibration method has been put forward. Taken scattering coefficient, number density, and other physical parameters into consideration, the visibility are given to each particle, making the boundary identification process starts from the same basis of Mie-Scattering experiments, which are widely used as calibration for spray modelling. The calibration method has been tested under cold spray condition, and could precisely fix the gap between raw simulation data and experimental data, proving its feasibility and necessity in CFD calibration especially under the high temperature conditions when the density difference between regions is apparent.

It should be noted that the point of this paper is not to prove the in-accuracy of simulation or experiment. The point of this paper is to put forward the issue that there exist gaps between experiments and simulation studies, and the usage of each other as calibration without any bridging would lead to wrong calibration results, which may affect the following investigations. The methodology presented in this paper is only feasible for Mie-Scattering based experiments, and common efforts should be made to validate other experimental methods form the physics basis, therefore, bridge up the gap between experiment and simulation.

\section{Nomenclature}

\begin{tabular}{cc}
\hline$r$ & Explanation \\
$\varphi$ & Distance between scattering event and camera \\
$\theta$ & Polarize angle, depending on refractive indexes \\
$\pi_{n}$ & Scattering angle \\
$\tau_{n}$ & First associated Legendre function \\
$m$ & Second associated Legendre function \\
$\alpha$ & Relative refractive index \\
$\varphi_{k}$ & $\pi \mathrm{D} / \lambda$ \\
$\varepsilon_{k}$ & Bessel function \\
$I_{0}$ & Hankel function \\
$n$ & Light intensity of single droplet \\
$I$ & Number of droplets in the parcel \\
\hline
\end{tabular}




\section{References}

[1] He, R., Yi, P., Li, T., Zhou, X., and Gu, Y., 2020, "EVALUATIONS OF THE KH-RT BREAKUP AND DYNAMIC STRUCTURE SGS MODELS FOR EVAPORATING SPRAYS UNDER DIESEL ENGINE-LIKE CONDITIONS," AAS, 30(3).

[2] Niu, Y.-Y., Wu, C.-H., Huang, Y.-H., Chou, Y.-J., and Kong, S.-C., 2021, "Evaluation of Breakup Models for Liquid Side Jets in Supersonic Cross Flows," Numerical Heat Transfer, Part A: Applications, 79(5), pp. 353-369.

[3] Fansler, T. D., and Parrish, S., 2005, "Spray Measurement Technology: A Review - IOPscience," Measurement Science and Technilogy, (26).

[4] Hung, D. L. S., Harrington, D. L., Gandhi, A. H., Markel, L. E., Parrish, S., Shakal, J. S., Sayar, H., Cummings, S. D., and Kramer, J. L., 2009, "Gasoline Fuel Injector Spray Measurement and Characterization - A New SAE J2715 Recommended Practice on JSTOR," SAE International Journal of Fuels and Lubricants, 1, pp. 534-548.

[5] Rachakonda, S. K., Wang, Y., Grover, R. O., Moulai, M., Baldwin, E., Zhang, G., Parrish, S., Diwakar, R., Kuo, T.-W., and Schmidt, D. P., 2018, "A Computational Approach to Predict External Spray Characteristics for Flashing and Cavitating Nozzles," International Journal of Multiphase Flow, 106, pp. 21-33.

[6] Mundy, W. C., Roux, J. A., and Smith, A. M., 1974, "Mie Scattering by Spheres in an Absorbing Medium*," J. Opt. Soc. Am., JOSA, 64(12), pp. 1593-1597.

[7] Zeng, W., Xu, M., Zhang, Y., and Wang, Z., 2013, "Laser Sheet Dropsizing of Evaporating Sprays Using Simultaneous LIEF/MIE Techniques," Proceedings of the Combustion Institute, 34(1), pp. 1677-1685.

[8] Linne, M., 2013, "Imaging in the Optically Dense Regions of a Spray: A Review of Developing Techniques," Progress in Energy and Combustion Science, 39(5), pp. 403-440.

[9] "MiePlot" [Online]. Available: http://www.philiplaven.com/mieplot.htm. [Accessed: 28-Feb-2021].

[10] Gao, Y., Wu, S., Dong, X., Li, X., and Xu, M., 2019, "Evaporation and Atomization Characteristics of Dual-Fuel System under Flash Boiling Conditions," Applied Thermal Engineering, 161, p. 114161.

[11] Beale, J. C., and Reitz, R. D., 1999, "MODELING SPRAY ATOMIZATION WITH THE KELVINHELMHOLTZ/RAYLEIGH-TAYLOR HYBRID MODEL," AAS, 9(6). 